\title{
Stability of the different arms of a DNA tetrahedron and its interaction with a minor groove ligand
}

\author{
Michael L. J. Carter, ${ }^{a}$ David A. Rusling ${ }^{a}$, Steven Gurr ${ }^{a}$, Tom Brown ${ }^{b}$ and Keith R. Fox ${ }^{a}$ \\ a School of Biological Sciences, University of Southampton, Southampton, United Kingdom, \\ SO17 1BJ. \\ ${ }^{b}$ Department of Chemistry, University of Oxford, Oxford, United Kingdom, OX1 3TA.
}

Keywords: DNA tetrahedron, Hoechst 33258, minor groove ligand, nanostructure,

\section{Research Highlights}

- All the edges of a DNA tetrahedron melt at the same temperature.

- Hoechst 33258 stabilises a DNA tetrahedron by binding to its AATT recognition sequence.

- Stabilisation propagates throughout the tetrahedron which melts as a single entity

\section{ABSTRACT}

DNA strands can be designed to assemble into stable three-dimensional structures, based on Watson-Crick base pairing rules. The simplest of these is the DNA tetrahedron that is composed of four oligonucleotides. We have redesigned the sequence of a DNA tetrahedron so that it contains a single (AATT) binding site for the minor groove binding ligand Hoechst 33258 . We examined the stability of this structure by placing fluorescent groups within each of its edges and have shown that all the edges melt at the same temperature in the absence of the ligand. The minor groove ligand still binds to its recognition sequence within the tetrahedron and increases the melting temperature of the folded complex. This ligand-induced stabilisation is propagated into the adjacent helical arms and the tetrahedron melts as a single entity in a cooperative fashion 


\section{Introduction.}

Three-dimensional DNA nanostructures can be designed and assembled using Watson-Crick base-pairing rules [1-5]. These folded nanostructures have a number of potential applications such as drug delivery vehicles [6-12] and as tools for biosensing $[2,13-16]$. The simplest of these is the DNA tetrahedron, which can be formed from four oligonucleotides that containing complementary regions that assemble to form the six edges and four faces of the tetrahedron (Fig. 1A). The DNA tetrahedron, first described by Goodman et al. $[17,18]$ is comprised of four oligonucleotide strands, which, assemble to form a triangular based pyramid. In one design [18], each edge of the tetrahedron is comprised of a 20 base pair duplex and unpaired adenosines are placed at the corners, between the helical edges. Each corner contains a single unpaired base $(A)$ from each strand, and the ends of each strand come together to generate a nick at the centre of each helical edge. The minor groove ligand Hoechst 33258 is known to bind to AT-tracts of at least four bases in length and it binds with particularly high affinity to the sequence AATT [19-21]. We have re-designed the sequence of this tetrahedron so that it contains a single AATT binding site in the centre of one edge (as well as other unique sequences on the other edges) (Fig. 1A) and have examined how ligand binding to this site affects the stability of each part of the folded structure.
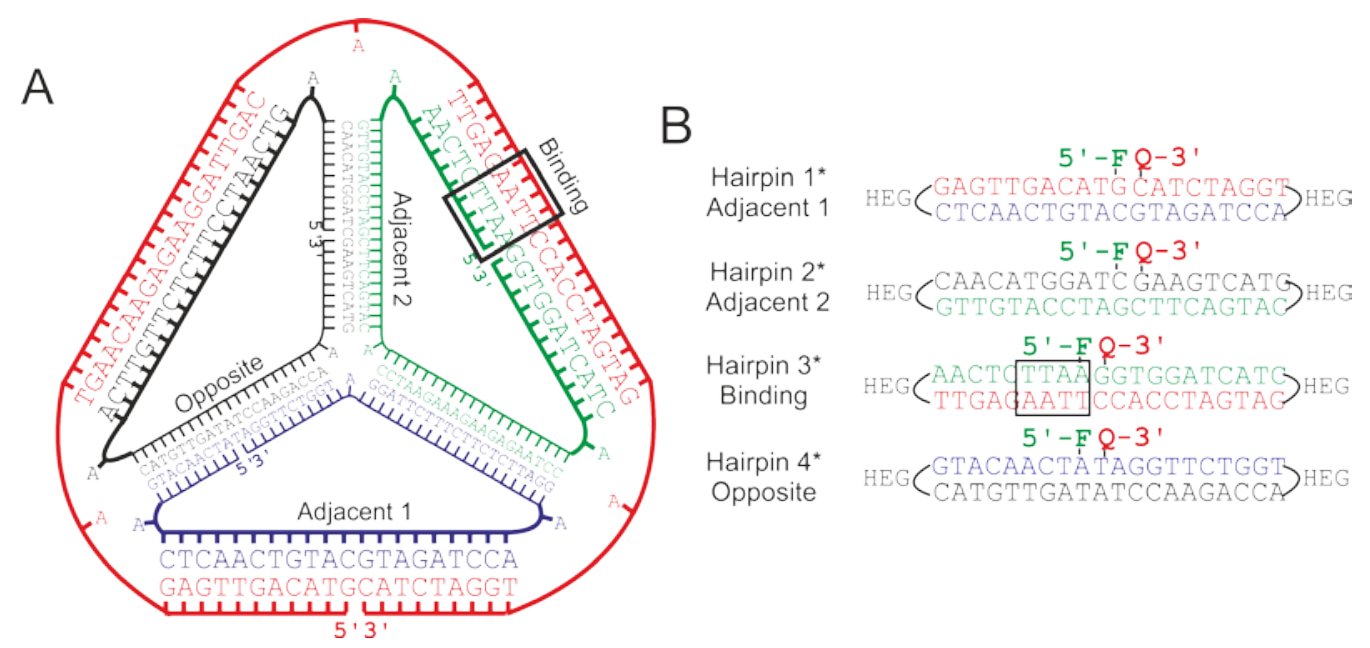

Fig. 1. (A) A schematic of the four oligonucleotide strands which make up the tetrahedron. Each strand is shown in a different colour. The AATT binding site for Hoechst 33258 is boxed. (B) Sequence of the fluorescently labelled hairpin oligonucleotides that correspond to each edge of the tetrahedron. HEG = hexaethylene glycol, $\mathrm{F}=$ fluorescein, $\mathrm{Q}=$ dabcyl. 


\section{Materials and Methods}

\subsection{Oligonucleotides and Ligands}

Oligonucleotides were obtained from ATDBio Ltd. Their sequences are shown in Table S1. Hoechst 33258 was purchased from Sigma-Aldrich (Poole, UK), dissolved in DMSO at a stock concentration of $10 \mathrm{mM}$ and diluted to working conditions in aqueous buffers immediately before use.

\subsection{Radiolabelling}

Oligonucleotides were 5'-end labelled with ${ }^{32} \mathrm{P}$ using $\mathrm{Y}^{3}{ }^{32} \mathrm{P}-[\mathrm{ATP}]$ and polynucleotide kinase. The labelled oligonucleotides were purified on $9.6 \%$ denaturing polyacrylamide gels, containing $8 \mathrm{M}$ urea. The labelled bands were excised from the gel and eluted overnight into $10 \mathrm{mM}$ Tris- $\mathrm{HCl}$ pH7.5 containing $10 \mathrm{mM}$ EDTA. The eluted DNA was precipitated with ethanol and dissolved in $10 \mathrm{mM}$ Tris- $\mathrm{HCl}$ pH 7.5 containing $0.1 \mathrm{mM}$ EDTA at a concentration of about 10 counts per second per microlitre, as determined on a hand-held Geiger counter.

\subsection{Tetrahedron and duplex assembly}

The four oligonucleotides that make up the tetrahedron were mixed at a concentration of $1 \mu \mathrm{M}$ each in $50 \mathrm{mM}$ sodium phosphate buffer $\mathrm{pH} 7.4$. The mixture was heated at $95^{\circ} \mathrm{C}$ for 5 minutes then cooled quickly on ice. Duplexes corresponding to each of the four oligonucleotide strands that make up the DNA tetrahedrons were annealed in the same fashion. For experiments with radiolabelled oligonucleotides, the DNA tetrahedron mixture was doped with the relevant 5' end radiolabelled oligonucleotide before annealing.

Successful formation of the tetrahedron was confirmed by agarose gel electrophoresis of the mixture alongside one, two and three stranded combinations of the constituent oligonucleotides as shown in Fig. S1.

\subsection{Reaction with diethylpyrocarbonate (DEPC)}

$5 \mu \mathrm{LEPC}$ was added to $10 \mu \mathrm{l}$ of $1 \mu \mathrm{M}$ annealed DNA tetrahedron or duplex DNA samples in $50 \mathrm{mM}$ sodium phosphate buffer $(\mathrm{pH} \mathrm{7.4)}$ and incubated for 20 minutes at room temperature. The reaction was stopped by ethanol precipitation, followed by washing with 70 $\%$ ethanol and the DNA pellet was dried under vacuum. The DNA was re-dissolved in $20 \mu \mathrm{l}$ $10 \%(\mathrm{v} / \mathrm{v})$ piperidine and heated at $95^{\circ} \mathrm{C}$ for 30 minutes. The samples were then dried under vacuum, washed twice with $20 \mu \mathrm{l}$ water, re-dried and dissolved in $5 \mu \mathrm{l}$ formamide, containing $10 \mathrm{mM}$ EDTA and bromophenol blue. The solution was heated at $95{ }^{\circ} \mathrm{C}$ for 5 minutes, crash cooled on wet ice and loaded onto a $40 \mathrm{~cm} 12 \%$ denaturing polyacrylamide gel. 


\subsection{UV melting}

Equimolar amounts of the four oligonucleotides were diluted in $1 \mathrm{ml} 50 \mathrm{mM}$ sodium phosphate buffer $(\mathrm{pH} 7.4)$ so that the sample had an $\mathrm{OD}_{260}$ of 0.5 . The samples were annealed by heating at $95{ }^{\circ} \mathrm{C}$ for 15 minutes, then crash cooled on wet ice. For samples with Hoechst 33258, the ligand was added after annealing the DNA. Samples were transferred to $1 \mathrm{ml}$ quartz cuvettes and loaded onto a Cary 4000 UV-Vis spectrophotometer (Agilent Technologies). Experiments were repeated in duplicate or triplicate. Samples were first heated to $80^{\circ} \mathrm{C}$ at a rate of $1{ }^{\circ} \mathrm{C} / \mathrm{min}$ followed by cooling to $20^{\circ} \mathrm{C}$, also at $1^{\circ} \mathrm{C} / \mathrm{min}$. $T_{m}$ values were obtained from the first derivative of the melting curves.

\subsection{Fluorescence melting}

Oligonucleotides, modified with a 5'-carboxyfluorescein (FAM) and 3'-dabcyl, were used to measure $T_{m}$ values of the DNA tetrahedron and compared with labelled hairpin duplexes corresponding to the same duplex edge (Fig. 1B). The oligonucleotides were diluted to $1 \mu \mathrm{M}$ in $50 \mathrm{mM}$ sodium phosphate buffer $(\mathrm{pH} \mathrm{7.4)}$ and mixed in a total volume of $20 \mu \mathrm{l}$. The DNA was annealed by heating to $95^{\circ} \mathrm{C}$ for 5 minutes then crash cooled on wet ice. Hoechst 33258 was added to DNA after annealing. Fluorescence melting curves were determined using a Roche LightCycler®. Samples were heated to $95^{\circ} \mathrm{C}$ at a rate of $5^{\circ} \mathrm{C} / \mathrm{min}$, held at 95 ${ }^{\circ} \mathrm{C}$ for 5 minutes, cooled to $30^{\circ} \mathrm{C}$ at a rate of $5{ }^{\circ} \mathrm{C} / \mathrm{min}$, held at $30^{\circ} \mathrm{C}$ for 5 minutes and the heated to $95{ }^{\circ} \mathrm{C}$ again at a rate of $5{ }^{\circ} \mathrm{C} / \mathrm{min}$. We observed no differences between the melting and annealing curves. All samples were run in triplicate.

\section{Results and Discussion}

\subsection{Tetrahedron assembly and stability}

We first demonstrated that this sequence, which is adapted from the one used by Goodman et al., still folds to form a tetrahedron, by examining its retardation on agarose gels (Fig. S1). As the different strands are combined, the mobility decreases and the species with all four strands has the lowest mobility. We also examined the reaction of the complete structure with diethylpyrocarbonate (DEPC) (Fig. S2). DEPC reacts at $\mathrm{N} 7$ of exposed adenines $[22,23]$ and, as expected, it can be seen that this is especially reactive at the unpaired adenines in the corners of the structure, as indicated by the asterisks.

UV-melting experiments with the complete tetrahedron (Fig. 2A) show that this melts with a $T_{m}$ of about $46{ }^{\circ} \mathrm{C}$ (Table 1 ). This corresponds to the global melting of the entire structure, but does not provide any information on the stability of the individual 
edges. We therefore incorporated fluorescein and dabcyl at the 5'- and 3'-ends of each oligonucleotide in turn, and compared their melting transitions with hairpin oligonucleotides corresponding to the labelled edges, with fluorescein and dabcyl in similar positions (Fig. 1B). When the complex is folded these groups are in close proximity and the fluorescence is reduced by collisional quenching [24]; when the strands melt these groups are separated and there is a large increase in fluorescence. The results are shown in Fig. 2 B, C and Table 1. The melting profiles of the labelled tetrahedrons are very similar (Fig. 2B), and their $T_{m} s$ are not affected by the edge on which fluorophores are located. These also display similar $T_{m} \mathrm{~S}$ to that determined by UV-melting for the entire structure. The hairpin oligonucleotides melt at much higher temperatures than the tetrahedron, as expected, as they are intramolecular species. However, more importantly, they each have different characteristic melting temperatures; for example, hairpin 3 melts $12{ }^{\circ} \mathrm{C}$ lower than hairpin 1 . It therefore appears that, although each edge has its own characteristic melting temperature, in the context of the complete structure, the stability of each of edge is determined by the stability of the complete tetrahedron, and that each of the 20-mer duplex edges does not behave independently.
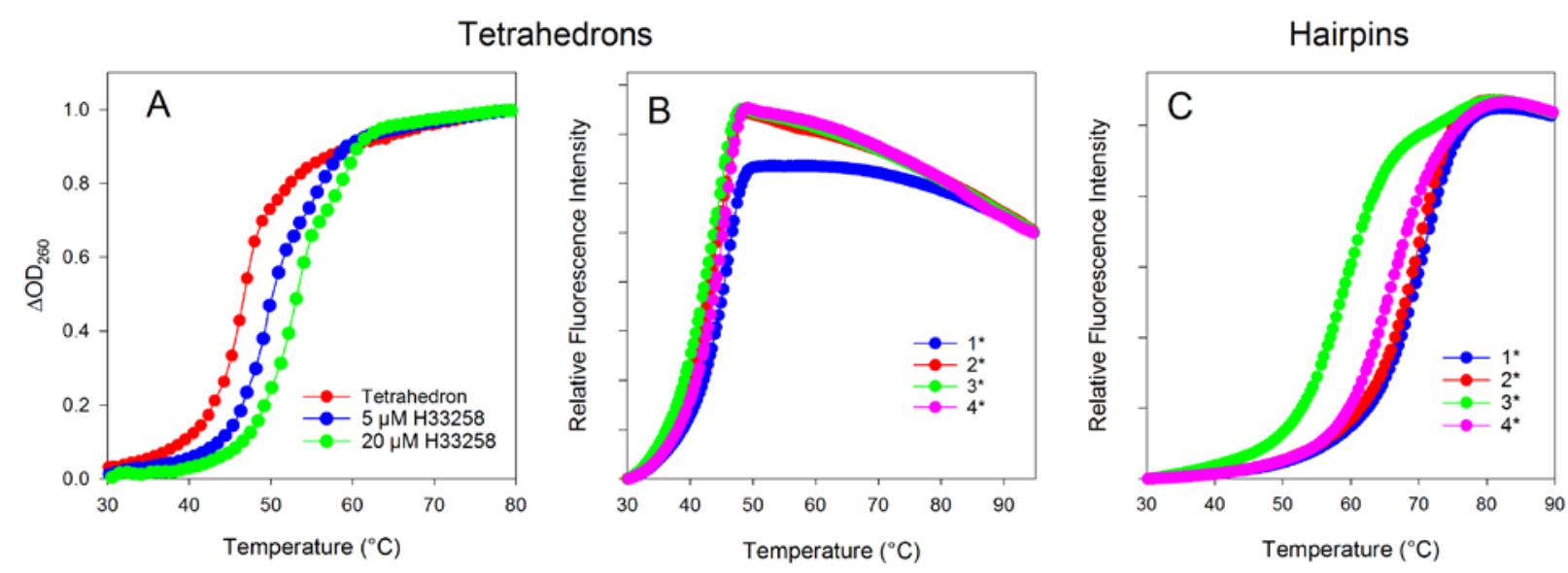

Fig. 2 Melting curves for the assembled tetrahedron. A) Melting curve of the tetrahedron determined by changes in the absorbance at $260 \mathrm{~nm}$ in the presence and absence of different concentrations of Hoechst 33258. B) Fluorescence melting curves for the tetrahedrons that have been labelled with fluorescein and dabcyl on each of the component strands in turn. C) Fluorescence melting curves for the hairpin oligonucleotides that correspond to each of the edges of the tetrahedron. 


\begin{tabular}{|c|c|c|c|c|c|c|c|c|c|}
\hline \multirow{3}{*}{$\begin{array}{c}\text { Hoechst } \\
(\mu \mathrm{M})\end{array}$} & \multirow{3}{*}{$\begin{array}{l}\text { UV } T_{m}\left({ }^{\circ} \mathrm{C}\right) \\
\text { Tetrahedron }\end{array}$} & \multicolumn{8}{|c|}{ Fluorescence $T_{m}\left({ }^{\circ} \mathrm{C}\right)$} \\
\hline & & \multicolumn{2}{|c|}{ Binding Edge } & \multicolumn{2}{|c|}{ Adjacent 1} & \multicolumn{2}{|c|}{ Adjacent 2} & \multicolumn{2}{|c|}{ Opposite } \\
\hline & & Hairpin & Tetrahedron & Hairpin & Tetrahedron & Hairpin & Tetrahedron & Hairpin & Tetrahedron \\
\hline 0 & $46.3 \pm 0.0$ & $59.1 \pm 0.3$ & $44.3 \pm 0.1$ & $71.1 \pm 0.2$ & $46.1 \pm 0.6$ & $69.7 \pm 1.2$ & $45.0 \pm 0.1$ & $66.3 \pm 0.2$ & $45.9 \pm 0.1$ \\
\hline 5 & $49.3 \pm 0.0$ & $65.7 \pm 0.2$ & $46.9 \pm 0.1$ & $71.2 \pm 0.4$ & $46.7 \pm 0.1$ & $69.2 \pm 0.8$ & $47.4 \pm 0.1$ & $66.2 \pm 0.1$ & $46.2 \pm 0.3$ \\
\hline 20 & $52.6 \pm 0.1$ & $67.2 \pm 0.2$ & 50.60 .3 & $71.7 \pm 0.3$ & $49.0 \pm 0.3$ & $69.6 \pm 0.8$ & $50.6 \pm 0.2$ & $66.5 \pm 0.4$ & $48.3 \pm 0.6$ \\
\hline
\end{tabular}

Table 1. Melting temperatures $\left(T_{m},{ }^{\circ} \mathrm{C}\right)$ of the tetrahedron and hairpin oligonucleotides with varying concentrations of Hoechst 33258, determined by UV and fluorescence melting experiments.

\subsection{Interaction with Hoechst 33258}

We were interested to discover whether DNA-binding agents are still able to interact with the constrained three-dimensional shape of the tetrahedron, and to examine their effect on the stability of the folded complex. This sequence was designed to contain a single Hoechst 33258 binding site (AATT) in the centre of one of the edges. Band-shift experiments demonstrated that $50 \mu \mathrm{M}$ Hoechst 33258 did not affect assembly of the tetrahedron, whether added before or after annealing of the four DNA strands (not shown). DNase I footprinting experiments, comparing the interaction of Hoechst 33258 with a tetrahedron containing ${ }^{32} \mathrm{P}$-labelled oligo 1 and a duplex with its complementary strand, are shown in Fig. 3. These show clear footprints at the AATT binding site on both the simple duplex and the tetrahedron, with a similar concentration dependence. As expected, no clear footprints are evident on any of the other strands, as these do not contain Hoechst 33258 binding sites. The highest ligand concentration generates weak attenuations in the oligopyrimidine tracts of oligo 4, which are evident on both the duplex and tetrahedron. These experiments confirm that the interaction of Hoechst 33258 with its AT-rich binding site is not affected by assembling this within a tetrahedron. 


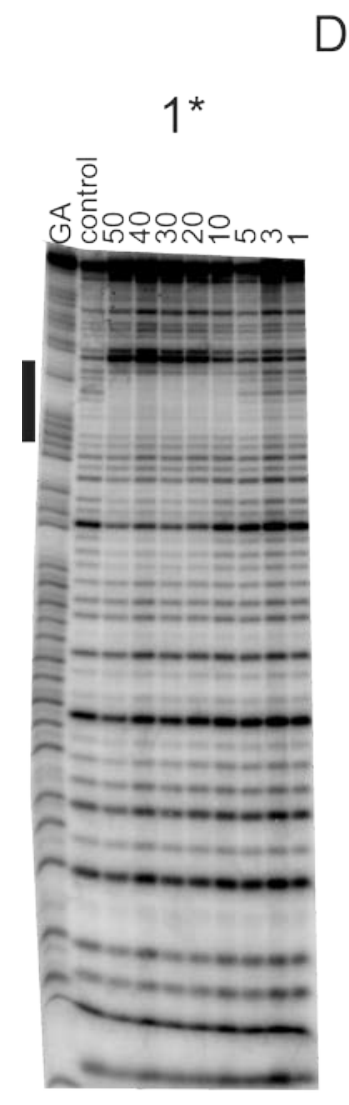

\section{Duplex}
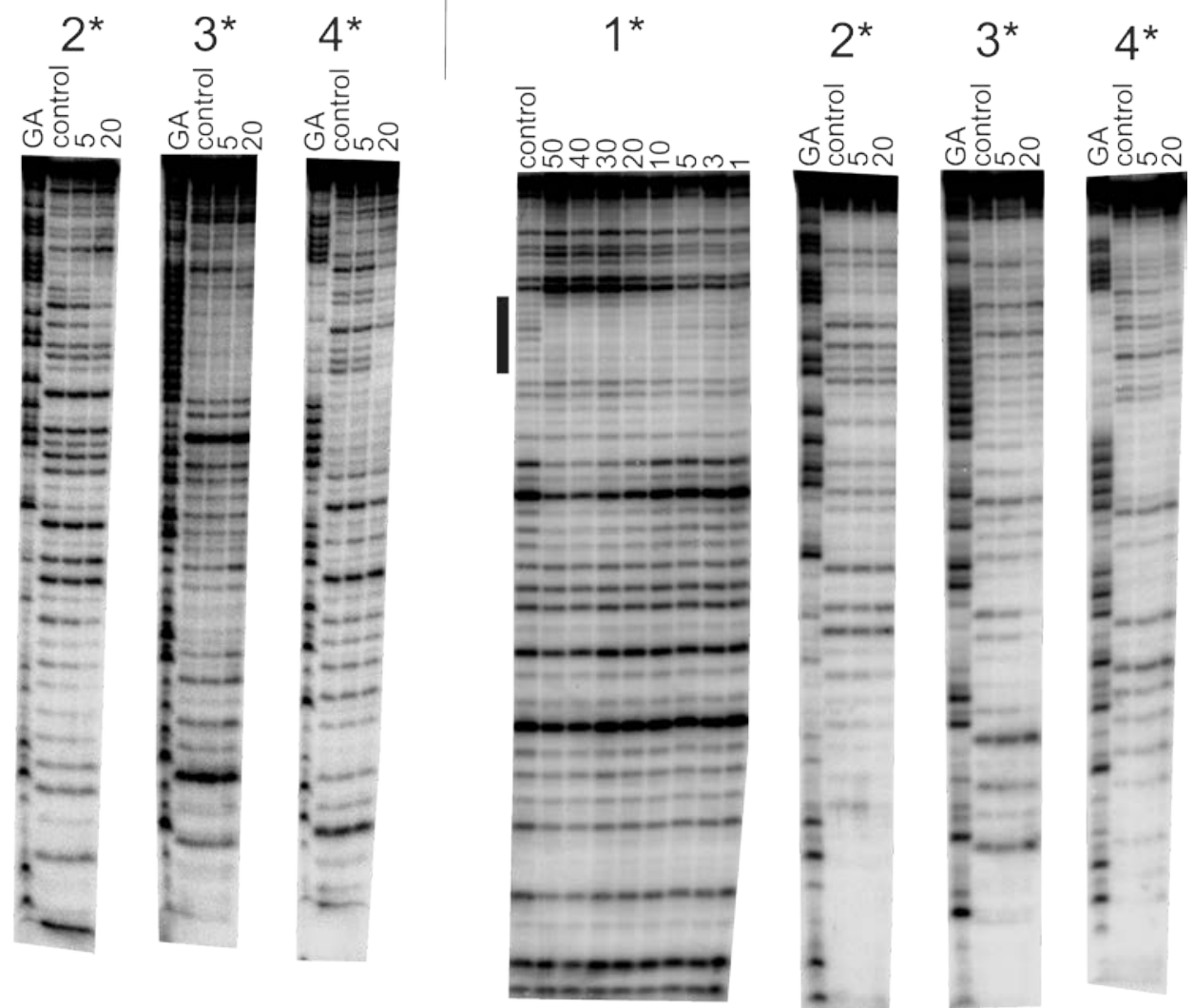

Fig. 3. DNase I footprinting gels for the interaction of Hoechst 33258 with the folded tetrahedron that was labelled on each of the strands in turn or with a fully complementary duplex generated with the labelled strand. The ligand binding site (AATT) is indicated by the filled bar alongside the labelled sequence 1 . Hoechst concentrations $(\mu \mathrm{M})$ are shown at the top of each gel lane. Tracks labelled 'GA' are markers specific for purines

UV thermal melting curves on the complete tetrahedron in the presence and absence of Hoechst 33258 are shown in Fig. 2A and the $T_{m}$ values are presented in Table 1. These show clear stabilisation of the DNA tetrahedron with an increase of $6{ }^{\circ} \mathrm{C}$ in the presence of $20 \mu \mathrm{M}$ ligand. However, these results do not provide any information on how the ligand affects specific regions of the tetrahedron. By placing fluorescein and dabcyl pairs on each edge of the tetrahedron in turn, we assessed how binding to the AATT site on one edge affects the stability of the other edges. In each case we compared the effect of Hoechst 33258 on a labelled tetrahedron with its effect on the hairpin oligonucleotide corresponding to the sequence of the same edge. The results are shown in Fig. 4 and are summarised in Table 1 . The results with the hairpin sequences clearly show that only the one containing the ligand binding site (AATT) shows any significant changes in the presence of the ligand. In contrast, when the different edges of the tetrahedron were studied, all the beacon edges showed some stabilisation, with the greatest effect seen with the one containing the AATT site. Note 
that the $T_{m}$ values of the differently labelled tetrahedrons are similar, even though their constituent duplexes show different melting properties. It therefore appears that interaction of the ligand with one edge of the tetrahedron increases the stability of the other edges, and that the complex melts in a cooperative fashion as a single unit.
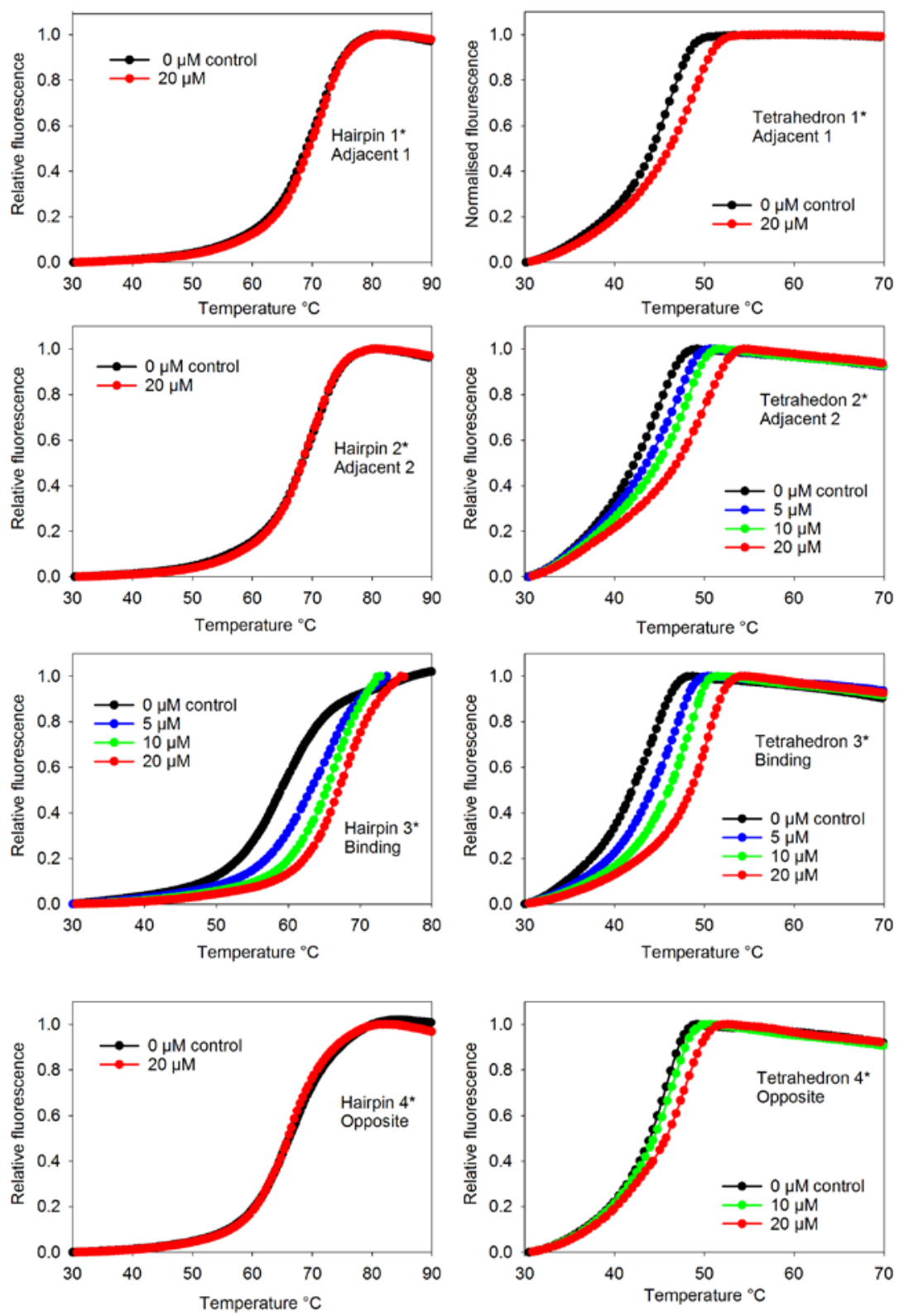

Fig. 4. Fluorescence melting curves for the assembled tetrahedron and the hairpin oligonucleotides in the presence of Hoechst 33258.

\section{Conclusions}

By using fluorescently-labelled oligonucleotides we have been able to assess the stability of different regions of a folded DNA nanostructure. As far as we are aware this 
is the first time that this method has been used to assess stability at specific locations within a DNA nanostructure. The results demonstrate that the tetrahedron melts as a single structure and that the stability of each vertex is determined by the entire structure and not just be the local sequence. We have also investigated the interaction of the sequence-specific minor groove binding ligand Hoechst 33258 with a DNA tetrahedron that contains a single AATT binding site on one of its edges. DNase I footprinting confirmed that this ligand can still access its binding site within the assembled tetrahedron, with no significant changes in affinity. UV melting studies showed that the ligand can globally stabilise this nanostructure, while the use of fluorescent beacons strategically placed throughout the structure has demonstrated ligand-induced stability across the entire structure, which melts cooperatively with a single transition. This type of behaviour, in which ligand binding to one region affects the stability of adjacent (non-bound) regions has been previously reported for simple linear duplexes, based on blocks of repeating nucleotides [25], but this is the first report of this effect for a DNA nanostructure in which an increase in stability is extended across DNA junctions. Since ligand binding can affect adjacent areas across DNA junctions, these observations suggest that nanostructures will not necessarily behave in the same manner as linear DNA when interacting with small molecules or proteins.

\section{Acknowledgement}

MLJC was supported by an EPSRC PhD studentship 


\section{References}

[1] S. Modi, D. Bhatia, F.C. Simmel, Y. Krishnan, Structural DNA Nanotechnology: From Bases to Bricks, From Structure to Function, J. Phys. Chem. Lett., 1 (2010) 1994-2005.

[2] H. Pei, X.L. Zuo, D. Zhu, Q. Huang, C.H. Fan, Functional DNA Nanostructures for Theranostic Applications, Acc. Chem. Res., 47 (2014) 550-559.

[3] A.V. Pinheiro, D.R. Han, W.M. Shih, H. Yan, Challenges and opportunities for structural DNA nanotechnology, Nature Nanotechnology, 6 (2011) 763-772.

[4] N.C. Seeman, DNA in a material world, Nature, 421 (2003) 427-431.

[5] N.C. Seeman, Nanomaterials Based on DNA, Annu. Rev. Biochem., 79 (2010) 65-87.

[6] M. Chang, C.S. Yang, D.M. Huang, Aptamer-Conjugated DNA Icosahedral Nanoparticles As a Carrier of Doxorubicin for Cancer Therapy, ACS Nano, 5 (2011) 6156-6163.

[7] S.M. Douglas, I. Bachelet, G.M. Church, A Logic-Gated Nanorobot for Targeted Transport of Molecular Payloads, Science, 335 (2012) 831-834.

[8] Q. Jiang, C. Song, J. Nangreave, X.W. Liu, L. Lin, D.L. Qiu, Z.G. Wang, G.Z. Zou, X.J. Liang, H. Yan, B.Q. Ding, DNA Origami as a Carrier for Circumvention of Drug Resistance, J. Am. Chem. Soc., 134 (2012) 13396-13403.

[9] K.R. Kim, D.R. Kim, T. Lee, J.Y. Yhee, B.S. Kim, I.C. Kwon, D.R. Ahn, Drug delivery by a self-assembled DNA tetrahedron for overcoming drug resistance in breast cancer cells, Chem. Commun., 49 (2013) 2010-2012.

[10] A.S. Walsh, H.F. Yin, C.M. Erben, M.J.A. Wood, A.J. Turberfield, DNA Cage Delivery to Mammalian Cells, ACS Nano, 5 (2011) 5427-5432.

[11] G.Y. Zhang, Z.Y. Zhang, J.E. Yang, DNA Tetrahedron Delivery Enhances DoxorubicinInduced Apoptosis of HT-29 Colon Cancer Cells, Nanoscale Research Letters, 12 (2017).

[12] Y.X. Zhao, A. Shaw, X.H. Zeng, E. Benson, A.M. Nystrom, B. Hogberg, DNA Origami Delivery System for Cancer Therapy with Tunable Release Properties, Acs Nano, 6 (2012) 8684-8691.

[13] A. Kuzuya, Y. Sakai, T. Yamazaki, Y. Xu, M. Komiyama, Nanomechanical DNA origami 'single-molecule beacons' directly imaged by atomic force microscopy, Nat. Commun., 2 (2011) 449.

[14] P. Miao, B.D. Wang, X.F. Chen, X.X. Li, Y.G. Tang, Tetrahedral DNA NanostructureBased MicroRNA Biosensor Coupled with Catalytic Recycling of the Analyte, Acs Applied Materials \& Interfaces, 7 (2015) 6238-6243.

[15] H. Pei, N. Lu, Y.L. Wen, S.P. Song, Y. Liu, H. Yan, C.H. Fan, A DNA Nanostructurebased Biomolecular Probe Carrier Platform for Electrochemical Biosensing, Adv. Mater., 22 (2010) 4754-4758.

[16] Y.L. Wen, H. Pei, Y. Shen, J.J. Xi, M.H. Lin, N. Lu, X.Z. Shen, J. Li, C.H. Fan, DNA Nanostructure-based Interfacial engineering for PCR-free ultrasensitive electrochemical analysis of microRNA, Sci. Rep., 2 (2012) 867.

[17] R.P. Goodman, R.M. Berry, A.J. Turberfield, The single-step synthesis of a DNA tetrahedron, Chem. Commun., (2004) 1372-1373.

[18] R.P. Goodman, I.A.T. Schaap, C.F. Tardin, C.M. Erben, R.M. Berry, C.F. Schmidt, A.J. Turberfield, Rapid chiral assembly of rigid DNA building blocks for molecular nanofabrication, Science, 310 (2005) 1661-1665.

[19] A. Abu-Daya, P.M. Brown, K.R. Fox, DNA sequence preferences of several AT-selective minor groove binding ligands, Nucleic Acids Res., 23 (1995) 3385-3392.

[20] K.D. Harshman, P.B. Dervan, Molecular recognition of B-DNA by Hoechst 33258, Nucleic Acids Res., 13 (1985) 4825-4835. 
[21] M. Teng, N. Usman, C.A. Frederick, A.H.J. Wang, The molecular structure of the complex of Hoechst 33258 and the DNA dodecamer d(CGCGAATTCGCG), Nucleic Acids Res., 16 (1988) 2671-2690.

[22] J.G. McCarthy, L.D. Williams, A. Rich, Chemical-reactivity of potassium permanganate and diethylpyrocarbonate with B DNA - Specific reactivity with short A-tracts, Biochemistry, 29 (1990) 6071-6081.

[23] N.J. Leonard, J.J. McDonald, R.E.L. Henderson, M.E. Reichman, Reaction of diethylpyrocarbonate with nucleic acids components - adenosine, Biochemistry, 10 (1971) 3335-3342.

[24] R.A.J. Darby, M. Sollogoub, C. McKeen, L. Brown, A. Risitano, N. Brown, C. Barton, T. Brown, K.R. Fox, High throughput measurement of duplex, triplex and quadruplex melting curves using molecular beacons and a LightCycler, Nucleic Acids Res., 30 (2002) e39.

[25] J.F. Burd, R.M. Wartell, J.B. Dodgson, R.D. Wells, Transmission of stability (telestability) in deoxyribonucleic-acid - physical and enzymatic studies on duplex block polymer $d\left(C_{15} A_{15}\right) \cdot d\left(T_{15} G_{15}\right)$, J. Biol. Chem., 250 (1975) 5109-5113.

\section{TABLE OF CONTENTS GRAPHIC}

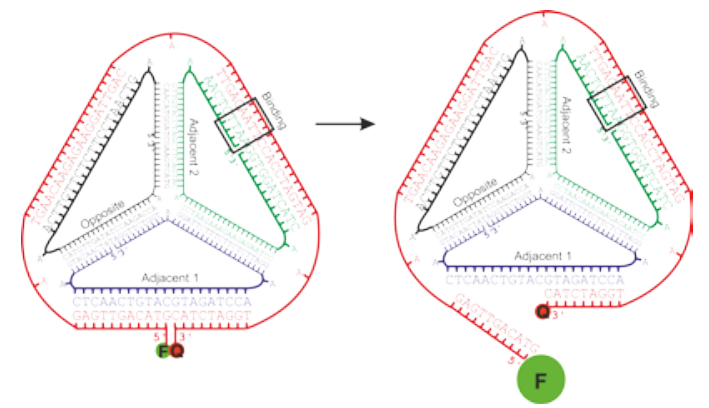

